Archives

\title{
La banque de données Médoc mécanisation de la France $\mathrm{XIX}^{\mathrm{e}}-\mathrm{XX}^{\mathrm{e}}$ siècles
}

\section{Serge Benoit et Gérard Emptoz}

\section{OpenEdition Journals}

\section{Édition électronique}

URL : http://journals.openedition.org/ccrh/2680

DOl : $10.4000 /$ ccrh. 2680

ISSN : $1760-7906$

Éditeur

Centre de recherches historiques - EHESS

Édition imprimée

Date de publication : 14 octobre 1995

ISSN : 0990-9141

\section{Référence électronique}

Serge Benoit et Gérard Emptoz, «La banque de données Médoc mécanisation de la France XIXe-XXe siècles », Les Cahiers du Centre de Recherches Historiques [En ligne], 14-15| 1995, mis en ligne le 27 février 2009, consulté le 01 mai 2019. URL : http://journals.openedition.org/ccrh/2680 ; DOI : $10.4000 /$ ccrh. 2680

Ce document a été généré automatiquement le 1 mai 2019.

Article L.111-1 du Code de la propriété intellectuelle. 


\section{La banque de données Médoc mécanisation de la France $\mathrm{XIX}^{\mathrm{e}}-\mathrm{XX}^{\mathrm{e}}$ siècles}

Serge Benoit et Gérard Emptoz

\section{Définition d'une problématique : la mécanisation envisagée comme l'un des processus centraux de l'industrialisation}

1 La mécanisation est l'un des aspects clés pour comprendre le processus d'ensemble de l'industrialisation. Cette notion recouvre en réalité deux dynamiques à la fois conjointes et distinctes. D'une part, la mécanisation comme substitution toujours plus étendue du machinisme au travail manuel dans la sphère de la production. De l'autre, comme extension continue des applications de la force mécanique inanimée - fournie par des sources d'énergie toujours plus diversifiées - en remplacement des énergies humaine et animale.

2 Par sa généralité et sa portée unifiante, la notion de mécanisation permet, en effet, de dépasser la diversité des branches et des secteurs pour accéder à des dynamiques qui introduisent au coeur même du phénomène. Ajoutons qu'elle est un ressort qui permet de cerner l'industrialisation comme un processus global, puisqu'aussi bien sa généralisation - consubstantielle à l'industrialisation elle-même -, ne concerne pas le seul domaine, assurément central, de l'industrie proprement dite, le champ manufacturier, mais s'étend à terme à l'ensemble des aspects de la culture matérielle, incluant outre les transports, la construction et l'agriculture, la communication sociale, la vie quotidienne, les loisirs, et jusqu'aux supports et auxiliaires de la production intellectuelle et artistique ${ }^{1}$

3 Il reste qu'à l'origine de ce processus de mécanisation, se place comme facteur décisif l'avènement, entre la fin du xviII ${ }^{\mathrm{e}}$ siècle et le début du suivant, d'un secteur à part entière 
de l'industrialisation, qui a comme caractéristique d'en être plus qu'une composante, mais l'une des conditions nécessaires de son développement : la construction mécanique lourde, encore appelée aujourd'hui secteur des biens d'équipement. Les déséquilibres insupportables à long terme auxquels ont abouti les priorités excessives données au secteur appelé Secteur A ou Secteur I (production des biens de production) de la problématique marxiste dans les expériences historiques des pays dit du «socialisme réel ", doivent être considérés comme la manifestation, sous une forme relevant de la pathologie économique, d'une nécessité universellement reconnue dans l'analyse de l'industrialisation, quant au rôle stratégique de la construction mécanique comme industrie industrialisante par excellence. Inversement, il est permis de se demander si la logique dite « des créneaux », qui a prévalu en France durant les années 1970 notamment, en laissant se poursuivre le démantèlement en longue durée de ce secteur, n'a pas fait abstraction du fait que celui-ci n'était précisément pas un secteur comme les autres. De même, la brève tentative de caractère volontariste pour tenter d'en enrayer le déclin entre 1982 et 1985 a peut-être en partie échoué parce qu'elle en avait oublié la spécificité au milieu du tissu industriel pour le traiter comme les autres activités frappées par la crise. On ne saurait trop insister, par delà ces vicissitudes de la politique industrielle française du dernier quart de siècle, postérieurement aux Trente Glorieuses, sur l'importance des conséquences qu'a comportées, dans un cadre de développement industriel resté national pour l'essentiel, la disparition de la majeure partie de la mécanique lourde française : celle-ci signifie, en effet, la perte de l'un des instruments fondamentaux permettant de maitriser le rythme et le contenu de l'industrialisation. Au delà de tous les débats, il n'est cependant pas douteux qu'il y ait là l'une des causes fondamentales de l'affaiblissement industriel français enregistré depuis le milieu des années 1970, sinon même de l'incapacité de l'économie nationale, depuis ce moment - plus qu'à opérer une reconquête - à retrouver durablement le chemin de la croissance.

4 Il n'est donc pas nécessaire de souligner davantage l'importance d'une étude systématique, faisant appel à des techniques quantitatives, de l'histoire de la construction mécanique comme secteur stratégique de l'industrialisation. Ajoutons qu'une telle approche est de nature, par son objet même, à éviter l'écueil d'une vision par trop française, et donc "provinciale » à l'échelle mondiale, du problème, en raison du caractère par excellence international, non seulement du développement technologique industriel depuis le siècle dernier - aspect quant à lui non discutable des travaux d'un David S. Landes -, mais de ce secteur-ci tout particulièrement. On mesure, en effet, de plus en plus la continuité, la multiplicité et l'extension des transferts de technologie intervenus dans le processus français de l'industrialisation depuis deux siècles, qui, en outre, ne furent pas unilatéraux. Car, s'il n'est pas contestable qu'à plusieurs phases, ces mouvements se soient principalement effectués vers la France à partir des pays étrangers initiateurs, c'est aussi de la France qu'à plus d'une reprise, sur certains autres aspects, vint l'innovation. Celle-ci, en fin de compte, est bien partie d'une pluralité, tout à la fois permanente et variable dans son contenu, de foyers internationaux, à l'opposé du schéma anglocentriste ayant longtemps prévalu dans l'historiographie dominante à propos du XIX e siècle.

5 C'est ce cadre d'ensemble qui a légitimé le projet, à l'initiative et sous la direction de Louis Bergeron, de constituer au sein du CRH une base de données automatisée à propos de la mécanisation, à partir du vaste domaine de la construction mécanique. 


\section{Les matériaux de base de la banque}

6 La possibilité et l'opportunité d'engager une telle entreprise sont apparues, il y a moins d'une dizaine d'années, comme moyen de capitaliser et de valoriser l'acquis documentaire formé par l'existence de plusieurs fichiers manuels se rapportant à cette thématique, de formation soit récente, soit plus ancienne. Sans jamais perdre de vue le caractère global de la notion de mécanisation, tel qu'il a été rappelé plus haut, la nature des études existantes a conduit à privilégier, dans la constitution de cet outil, plusieurs aspects relevant de ce que l'on peut appeler le "premier cercle», du processus d'industrialisation, c'est-à-dire les manifestations de la mécanisation dans la sphère de la production manufacturière.

\section{Les matériaux relatifs au thème énergétique}

7 Un premier ensemble de matériaux traités dans le cadre de la banque se rapporte, de fait, aux aspects énergétiques de la mécanisation.

Il s'est agi, initialement, en ce qui concerne l'apport propre du CRH, des fichiers issus de trois enquêtes collectives successives - dont deux réalisées avec l'aide du CNRS -, menées, à chaque fois sous l'égide du Centre et sous la conduite de Louis Bergeron, autour du thème du moteur hydraulique au XIX ${ }^{\mathrm{e}}$ siècle, d'abord dans une perspective d'archéologie industrielle en 1981-1982, puis d'histoire des sciences et des techniques de 1983 à $1986^{2}$.

Il n'y a pas lieu de revenir davantage ici sur ce qui a constitué le noyau initial de la banque, ni sur la problématique historique qui l'a inspirée, et qui rattachait au processus de la mécanisation un vaste ensemble documentaire constitué autour du thème du moteur hydraulique comme l'une des composantes centrales de la voie française de l'industrialisation - une voie qui s'est avérée être, en fait, sous le rapport des bases énergétiques, celle d'une majorité de pays actuellement développés. Ces enquêtes, d'où sont issus les matériaux intégrés depuis dans le stock documentaire de la banque, ont été suffisamment relatées dans le précédent article paru sur cet instrument dans le numéro 3 des présents Cahiers, d'avril $1989^{3}$. Les fichiers relatifs au moteur hydraulique dépassaient largement ce champ étroitement spécialisé, dans la mesure où la très grande majorité des fabricants de cette catégorie d'équipements en France pratiquaient des productions polyvalentes, de manière à pouvoir contrôler le marché des installations d'usines complètes - que l'on n'appelait pas encore " clés en mains » : aussi bien, ce premier corpus ouvrait-il en fait une fenêtre sur l'ensemble du processus de la mécanisation.

Il s'y est adjoint d'autres fichiers, se recoupant en partie avec les précédents, qui avaient été constitués antérieurement - en l'espèce durant les années 1970-par une autre formation de recherche, en l'espèce le Centre de documentation d'histoire des techniques, implanté au CNAM, qui ont pu être agrégés à la banque à la faveur de l'existence d'accords antérieurs entre le CDHT et le CRH Ce second ensemble documentaire provient, pour une part, de l'enquête, menée à l'initiative de Maurice Daumas, sur les industries de la région parisienne: celle-ci comportait un pan entier spécifiquement consacré au secteur de la construction mécanique, dont la capitale n'a cessé d'être le plus important foyer en France depuis, en fait, la fin du XviII ${ }^{\mathrm{e}}$ siècle. Pour 
l'autre part, il dérive des travaux sur la machine à vapeur auxquels a attaché son nom un autre grand pionnier de l'histoire des techniques en France, Jacques Payen.

11 L'ensemble à partir duquel l'outil informatisé a été construit initialement comprend quatre séries de fichiers :

- fichier des inventeurs essentiellement constitué à partir du fonds des brevets proprement dits ${ }^{4}$;

- fichier des sources bibliographiques relatives aux acteurs de l'innovation;

- fichier biographique des dossiers individuels de synthèse élaborés à propos d'un échantillon d'inventeurs majeurs ;

- fichier des entrepreneurs et entreprises de construction mécanique principalement issu de l'enquête du CDHT sur la mécanique parisienne.

12 La mise en place technique de la banque de données, devenue opérationnelle à partir de 1987-1988, a, en outre, permis d'intégrer les fichiers d'autres enquêtes conduites dans le cadre du CRH, ou en liaison étroite avec les activités des chercheurs du Centre impliqués.

Eu égard à l'importance de la liaison entre la mécanisation et les problèmes généraux de l'utilisation de l'énergie dans l'industrialisation, la banque a été enrichie du corpus, provenant de la collecte effectuée par Gérard Emptoz parmi les fonds de l'Institut national de la Propriété industrielle (INPI) des brevets relatifs à la carbonisation du bois et à celle de la houille (plus connue sous le terme de cokéfaction), dont il est apparu qu'ils concernaient des domaines d'application autrement plus diversifiés que leur usage métallurgique d'ordinaire seul ou principalement pris en compte. Bien qu'il s'agisse de données relatives aux combustibles, celles-ci ont été intégrées à la banque dans la mesure où elles étaient homogènes, de par leur nature documentaire, avec celles réunies sur le thème de cet autre ensemble d'inventions relatif à l'utilisation de l'énergie hydraulique, et avaient fait l'objet d'un premier traitement manuel avec le même type de fiches. Au total, ce corpus, couvrant la période 1791-1880, comprend près de 150 brevets relatifs à la carbonisation du bois, et une centaine concernant celle de la houille. En outre, l'élaboration des combustibles provenant d'une opération de carbonisation, dont certains - comme le coke au milieu du siècle dernier dans les foyers de locomotives - ont été directement employés à la production de force motrice - a donné lieu elle aussi à une véritable mécanisation, alors qu'il s'agissait au départ d'un travail exclusivement accompli manuellement, dans le cadre de la méthode traditionnelle en meules mise en oeuvre aussi bien pour le coke que pour le charbon de bois. Cette recherche sur les combustibles s'est inscrite dans une continuité délibérée avec l'enquête collective qui avait été menée, de 1985 à 1988, sous la direction de Denis Woronoff (lui-même membre du CRH de 1987 à 1993), sur le thème Forges et Forêts, et qui avait suscité l'engagement, de la part des auteurs, d'une première recherche systématique sur les innovations relatives à l'élaboration et à l'utilisation du combustible végétal ${ }^{5}$.

Comme dans le cas des précédentes enquêtes relatives au moteur hydraulique, les brevets ont été complétés par le maximum de références bibliographiques - en termes de sources contemporaines - relatives à ceux-ci et à leurs inventeurs, en plus de la référence à la source fondamentale prise aux fonds de l'INPI : publications de brevets, descriptions de machines, de procédés, d'installations industrielles ou d'applications des innovations, notices biographiques ou nécrologiques sur les inventeurs et les industriels, mentions de participations ou de récompenses de ces derniers à des expositions nationales, internationales ou universelles, etc. 


\section{Les matériaux relatifs aux équipements productifs sectoriels} suivre la mécanisation dans le secteur d'entraînement par excellence de la croissance industrielle au siècle dernier, le textile : les archives, versées et classées depuis 1987, de la Société alsacienne de Constructions mécaniques (SACM). Cette grande firme mulhousienne est, non seulement, parfaitement représentative du processus de fabrications complémentaires, visant à la fourniture d'usines complètes dans ce secteur, avec une double spécialité majeure orientée sur les machines textiles - de tous types et pour toutes les fibres - et sur les équipements dits de mécanique générale, c'est-à-dire les moteurs primaires hydrauliques et à vapeur et les transmissions. De la liquidation, intervenue depuis 1986, des deux filiales de la firme axées sur les machines textiles (SACM-T) et sur la mécanique générale (SACM-M), sont issus deux fonds d'archives qui ont pu être l'un après l'autre sauvegardés et classés grâce à l'existence d'une structure originale et propre au milieu industriel de Haute-Alsace, le CERARE (Centre rhénan d'archives et de recherches économiques) ${ }^{6}$. Ce fonds constitue un observatoire exceptionnel pour suivre le développement industriel, non seulement à l'échelle de la France, mais à celle du continent européen, en raison de la place tenue par l'exportation dans l'activité de la firme ${ }^{7}$, et de sa position, attestée par de nombreux témoignages contemporains, de plus important constructeur de machines textiles d'Europe continentale - jusqu'au début du $\mathrm{xx}^{\mathrm{e}}$ siècle au moins, sinon au-delà - de fait longtemps seul à pouvoir rivaliser avec les grandes maisons britanniques des Midlands.

$\mathrm{Au}$ milieu de ce fonds, on a privilégié, en vue d'une première exploitation, les registres généraux de commandes de machines textiles, qui commencent en 1844 et forment une série pratiquement continue jusqu'en 1893, et couvrent donc complètement la période française de l'entreprise, sous sa forme en commandite et sous la raison sociale André Koechlin et Cie, antérieurement à sa transformation en société anonyme en 1872 sous le régime de la législation allemande. Pour diverses raisons, il a été convenu que les dépouillements entrepris s'en tiendraient, dans une première phase de la recherche, à cette époque de l'histoire de la firme. Ces registres peuvent être précisés, pour un certain nombre de catégories de machines, par d'autres séries de registres spécifiques (ainsi, pour les métiers à filer self-acting ou renvideurs, à partir de 1852, ou encore les métiers à filer continus à anneaux, ou ring throstle, dont la production débuta à Mulhouse en 1883). On rappellera ici que, par un dépouillement simplement manuel, les livres de commandes de la SACM avaient fait l'objet, pour les années 1900-1913, d'une exploitation très globale 
dans une étude due à Raymond Poidevin, dans le cadre de ses recherches sur les relations économiques franco-allemandes avant la Première Guerre mondiale ${ }^{8}$.

Des épaves des archives de la firme, versées plus anciennement et conservées aux Archives municipales de Mulhouse, viennent compléter, par ailleurs, ces deux très importants ensembles documentaires. Parmi celles-ci, on relève tout particulièrement, en liaison avec les problématiques de la banque, trois registres de commandes de la firme spécifiquement consacrés aux turbines hydrauliques construites sur les plans de la firme, dont deux complets et continus pour les années 1843-1856, et pour les années 1876-1893, et un registre partiel se rapportant aux décennies 1860 et $1870^{\circ}$. Ce dernier sous-ensemble se place à l'interface entre le thème de l'innovation technique et de sa diffusion (en l'espèce les turbines du type dit Jonval-Koechlin qui ont assuré le renom international de l'entreprise) ${ }^{10}$ et celui de la mécanisation.

En vue de permettre une exploitation la plus approfondie possible des données brutes recueillies dans les registres, celles-ci ont été complétées systématiquement au moyen d'informations extérieures en vue d'aboutir à une documentation homogène sur la clientèle. Il s'est agi notamment de l'identification précise des établissements - au sens de sites-auxquels étaient destinés les équipements commandés, les registres ne mentionnant parfois que le siège social ou le principal établissement des firmes clientes, dans une période où les entreprises textiles, notamment en Haute-Alsace, tendaient de fait à comporter non plus une, mais une pluralité d'unités de production. On a cherché, de même, à déterminer le plus exactement possible la branche d'activité concernée par les commandes (fibre: coton, laine, lin, soie; stade du cycle productif: filature, tissage, teinture, impression, etc.).

21 Depuis sa mise en route en 1987-1988 - il convient de le préciser - la banque n'a cessé de s'enrichir des résultats des recherches menées en liaison avec le séminaire animé régulièrement chaque année, dans le cadre du CRH, au titre du DEA d'Histoire des Techniques, par les auteurs de cette contribution, sous l'intitulé Technologies énergétiques et développement industriel, $\mathrm{XIX} \mathrm{X}^{\mathrm{e}} \mathrm{- \textrm {XX }} \mathrm{e}^{\mathrm{e}}$ siècles. ${ }^{11} \mathrm{Ce}$ séminaire de recherche a été le lieu privilégié de mise en perspective des travaux issus de la saisie des données.

\section{L'organisation technique des données : un instrument construit autour des acteurs}

La mise en place initiale de la banque de données, sur le plan technique, a été assurée, en 1987-1988, par un travail de définition de la grille d'analyse de base ${ }^{12}$ et d'installation des outils informatiques appropriés.

Le choix d'un logiciel s'est porté vers le système CDS/ISIS (en version micro-compatible, dite mini-micro, en version 1.0), mis gratuitement à la disposition des Universités par l'UNESCO, qui en détient la propriété, en vertu d'une licence concédée le 6 décembre 1985 ${ }^{13}$. L'installation technique de la banque a été assurée avec le concours du Centre de calcul de l'INRP, dans le cadre d'une convention de collaboration conclue entre ce dernier organisme et l'EHESS L'opération a bénéficié jusqu'en 1992 de l'appui financier de l'exDirection des Bibliothèques, des musées et de l'Information scientifique et technique (DBMIST), puis de la Mission de la Recherche au Ministère de l'Éducation Nationale.

24 La première grille d'analyse, élaborée en 1987-1988, avait été plus spécialement organisée autour du thème des inventeurs dans le domaine des moteurs hydrauliques, en vue de 
traiter les quatre ensembles de fichiers manuels indiqués plus haut. Quatre types de notices, correspondant à chacun de ces fichiers ont, par suite, été établies. Elles comportent chacune un champ de référence, servant à l'identification de la notice en fonction du fichier concerné, et un champ spécifique prenant en compte les types de données propres à chaque fichier.

Un certain nombre de types de données transversales se retrouvent d'un fichier à l'autre, assurant ainsi la possibilité de larges croisements. Il s'agit de données d'ordre :

- socioprofessionnel: mécanicien, ingénieur civil, ingénieur des Ponts et Chaussées, constructeur, etc. ;

- sectoriel, quant à la branche d'activité concernée : moteur hydraulique, machine à vapeur, machine-outil, machines textiles, etc. ;

- économique : nature de la rémunération dans le cas d'un personnage ; effectifs, chiffre d'affaires dans le cas d'une entreprise, etc.;

- technique, quant à la nature des types de moteurs hydrauliques concernés : turbine centrifuge, roue à admission interne, etc. ;

- géographique, enregistrant l'adresse du personnage concerné, en ayant soin de distinguer, le cas échéant, lorsqu'il s'agit d'un inventeur, sa domiciliation momentanée lors du dépôt de brevet (souvent faite à Paris), de son adresse réelle, c'est-à-dire du lieu d'exercice de son activité.

D'autres types de données sont propres à chaque fichier. Ainsi les notices relatives aux inventeurs distinguent-elles la nature du dépôt effectué : brevet ou certificat d'addition, et mentionnent-elles la présence éventuelle de documents iconographiques accompagnant le texte du brevet. Celles de nature biographique signalent les repères fondamentaux de la vie du personnage (dates et lieux de naissance, alliances), sa filière de formation (polytechnicien, centralien, etc.), ses distinctions : Légion d'Honneur, médaille à une exposition, etc., ou encore les domaines (définis par des mots-clés communs) concernés par ses écrits et publications.

Le lancement du programme consacré à la SACM, à partir de 1990-1991, a conduit à définir une nouvelle grille d'analyse spécifiquement adaptée au dépouillement des registres de commandes, dans la perspective d'une étude fine des marchés de l'entreprise. La première partie de la fiche a été conçue dans l'optique spécifique d'une analyse de la clientèle de la firme, c'est-à-dire des utilisateurs d'équipements produits par celle-ci. L'adresse du client a été soigneusement conservée en vue d'une cartographie automatisée. La souplesse du logiciel retenu a permis, en application de l'observation faite précédemment, de distinguer, le cas échéant, le siège social de l'acquéreur du site final d'installation des matériels commandés.

La référence du document, au delà de la cotation propre au CERARE, est fondamentalement donnée par la date de la commande figurant sur le registre. Parfois, celui-ci comporte l'indication de la date de livraison, mais il est clair que l'ensemble des commandes inscrites sur ces livres généraux correspondent à des commandes fermes de la part des clients, et qui ont été exécutées par le constructeur.

La seconde partie de la notice informatisée établie pour les équipements fabriqués par la firme mulhousienne vise à permettre la saisie du maximum d'informations techniques sur les matériels commandés. L'un des éléments les plus importants a été ici l'établissement, à partir de sondages étendus à travers les registres, d'un lexique de termes techniques normalisés désignant les types de machines figurant dans les registres. 
Des équivalences ont été déterminées dans le cas de doublons, cependant qu'il a fallu veiller à éviter les faux amis (ainsi sur des termes phonétiquement voisins tels que bobineur et bobinoir). Ce thesaurus, dont un extrait est présenté ici en annexe, comporte actuellement quelque 120 termes normalisés. La fiche enregistre le nombre d'exemplaires de chaque type de machines commandés, et, dans le cas d'équipements de filature, le nombre total de broches inclus dans la commande, lorsqu'il est mentionné. Les acquisitions de pièces détachées, fréquemment associées à des achats de matériels entiers, mais quelquefois aussi faites pour elles-mêmes, sont également prises en compte. Le champ disponible pour les indications libres sert en particulier à noter l'existence d'un plan, lorsqu'il en est conservé un correspondant à la commande.

L'unité et l'homogénéité de l'instrument sont permises par le choix, dans tous les cas, d'une entrée à partir des hommes, ou des entreprises - en d'autres termes des personnes physiques ou des personnes morales -, qu'il s'agisse d'inventeurs, de concepteurs, de constructeurs, ou d'utilisateurs, ou si l'on préfère d'un accès privilégié à travers les acteurs individuels ou collectifs, en amont comme en aval du processus innovatif associé à la mécanisation. Ainsi, dans le cas des équipements produits par la SACM, y a-t-il un double accès à ceux-ci à partir de leur constructeur et de leurs acquéreurs, les clients de la firme mulhousienne. Cette primauté accordée à la dimension humaine et sociale du développement industriel et technique est assurément l'approche qui n'a cessé de donner son unité et sa continuité aux enquêtes successives qui, depuis 1979, ont été poursuivies sous l'égide du CRH, et sous la direction scientifique de Louis Bergeron.

\section{Résultats et perspectives d'exploitation de l'instrument}

31 Depuis la mise en place de l'instrument, ce sont plus de 5000 notices qui ont été saisies et rentrées dans la banque. Sur cet ensemble, à ce jour, plus de 3000 (3 224) notices se rapportent au thème énergétique dans la large définition qui a été indiquée plus haut, plus de 200 aux combustibles, et près de 2000 (1947) concernent les livres de commandes de la SACM, et couvrent les années 1844 à 1860 incluse.

Les produits susceptibles d'être issus directement de la banque, par traitement automatisé des données, sont de trois ordres.

Le premier niveau, élémentaire, correspond à l'obtention de statistiques générales. C'est ce type d'exploitation qui a été réalisé d'emblée avec l'aide de l'instrument dans le cas des brevets relatifs à la carbonisation du bois et de la houille, avec les multiples croisements qu'il autorise à propos des inventeurs. Appliqué au fonds, qui n'avait fait l'objet jusque là que d'une exploitation manuelle, des brevets relatifs aux moteurs hydrauliques, ce traitement a permis de faire apparaitre de nouveaux éléments et des tendances qui n'avaient pu être repérées par les moyens précédemment utilisés ${ }^{14}$. Ce premier niveau d'analyse permet d'établir des tableaux généraux, de mettre en évidence des périodisations, de procéder à des comparaisons entre données statistiques (ainsi, le parallèle, très suggestif, entre les brevets relatifs à la carbonisation du bois et ceux portant sur la cokéfaction). La comparaison, d'une année sur l'autre, du nombre de commandes de machines textiles enregistrées par la firme mulhousienne, correspondant simplement ici au nombre de notices établies pour chaque année, est en elle-même des 
plus éclairantes ; il en va de même des notices individuelles établies pour les turbines du même constructeur (cf. diagramme en annexe).

Le second niveau d'exploitation concerne l'analyse des marchés des entreprises, à travers ici le cas de la SACM L'objectif est de dégager les structures de la clientèle, ses continuités comme ses renouvellements, sa fidélisation en fonction de la fréquence d'apparition d'un client déterminé. Le fichier de la clientèle a été organisé pour être pouvoir donner lieu à une périodisation et à une cartographie. L'analyse doit permettre de suivre la diffusion de l'innovation, en termes sectoriels et géographiques. Ce type d'exploitation est rendu possible dans la mesure où le logiciel retenu permet d'appeler simultanément plusieurs mots-clés. Ce type d'analyse sera analogue aux études réalisées aux États-Unis sur certains types de machines textiles, à l'instar de celle conduite outre-Atlantique à propos du premier type de métier à tisser automatique mis sur le marché, celui conçu et construit par la firme Draper.

L'analyse des marchés de machines textiles de la firme mulhousienne vise à en dégager les structures élémentaires du point de vue de :

- l'évolution générale de ses ventes au cours du temps;

- la répartition géographique de sa clientèle, en fonction d'une part de la distinction entre marchés intérieur et extérieur, et d'autre part du changement d'espace économique de rattachement de la firme entre 1871 et 1918, en relation avec l'application au cas présent de la problématique générale des espaces frontaliers ;

- l'identification des clients, en ce qui concerne l'importance de leurs achats, leur fidélité, etc. ;

- la répartition des ventes par branches d'activité (en l'espèce en fonction de la nature des fibres: coton, laine, soie, etc., et du stade concerné du cycle productif : filature, tissage, impression, etc.) ;

- la structure des ventes par catégories de machines (métiers à filer mule-jenny, self-acting, continus à anneaux dans le cas de la filature, etc.).

Une application particulière de cette étude des marchés, destinée à tester les capacités de la banque, devrait être pouvoir être mise en oeuvre très prochainement. Elle consistera à croiser le fichier des commandes de machines textiles avec celui des commandes de turbines, sur la période où les deux séries de registres existent en parallèle, c'est-à-dire de 1843 à 1856. Il devrait être possible, de la sorte, de mesurer l'étendue du phénomène des marchés liés dans l'activité de l'entreprise, et son impact sur celle-ci. Une telle étude fera ressortir jusqu'à quel point il a pu exister, au profit de l'entreprise, un marché autonome des turbines, indépendant des fournitures d'usines complètes, et qui représentait à l'époque une tendance tout à fait moderne, et son impact sur le développement des ventes de la firme ${ }^{15}$. Pour être pleinement probant, toutefois, une telle étude devrait être complétée, si le classement, une fois achevé, du fonds relatif à la mécanique générale en révèle la possibilité - voire une autre source - par un croisement avec les ventes de machines à vapeur de la firme : il est, en effet, indispensable de prendre en compte ici l'importance, déjà connue par d'autres canaux, de l'usage de la vapeur soit comme moteur exclusif, soit comme énergie auxiliaire ou d'appoint permanent vis-à-vis de l'hydraulique, dans les régions textiles formant les principales zones d'implantation commerciale de la firme mulhousienne, à commencer par la Haute-Alsace, avec ici la dualité des usines à vapeur de l'agglomération mulhousienne elle-même, et les usines, pour la plupart à force mixte, des vallées vosgiennes. 

lexique technique historique élaboré à l'occasion de la mise en place de la banque. L'exigence de conservation dans le lexique du terme technique le plus exact, jointe aux capacités du logiciel adopté, permet de repérer et de dater l'apparition d'un terme technique nouveau, correspondant à une invention, jusqu'à sa commercialisation effective et son emploi opérationnel dans l'industrie, et de mesurer, ainsi par rapport à la datation des brevets, le décalage entre le stade de l'invention et celui de l'innovation.

Enfin, la banque devrait déboucher sur la réalisation d'outils généraux, de type plus classique, mais qui sera facilitée par le rassemblement automatisé des données. Il pourra s'agir, au premier chef, d'un dictionnaire des mécaniciens français du siècle dernier - au moins centré sur le thème fédérateur initial des moteurs hydrauliques. Par la réunion des deux grilles d'analyse mises en oeuvre, un tel projet permettrait d'intégrer à la fois une analyse sérielle d'histoire des techniques mettant en lumière la contribution des uns et des autres au mouvement innovatif, une approche prosopographique dans une perspective d'histoire sociale des entrepreneurs et des innovateurs, et la prise en compte, sur le plan de l'histoire économique, de leur place respective dans la production et les marchés.

\section{Annexe 1 - Exemples de notices Inventeurs}

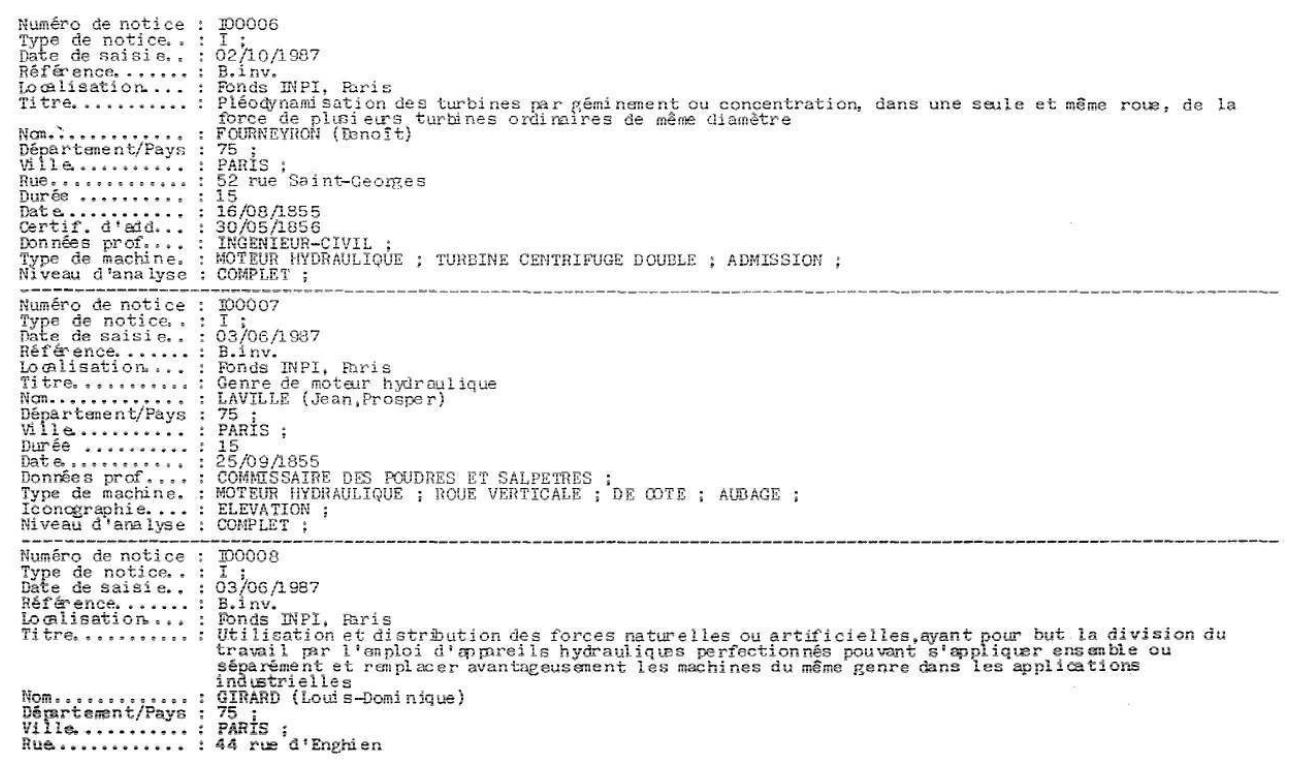


Annexe 2 - Exemples de notices Biographies

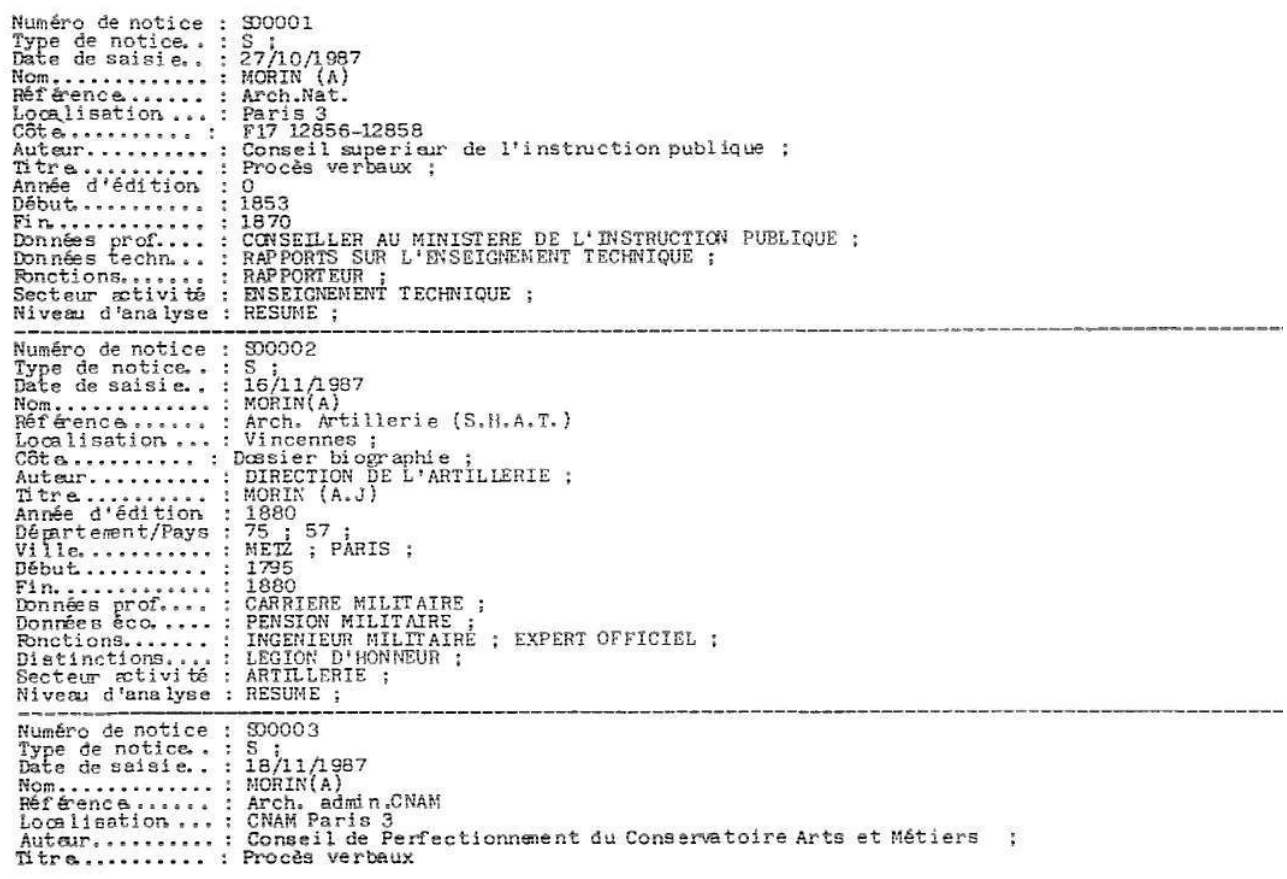

Annexe 3 - Exemple d'interrogation (partielle) à partir du nom BRAULT

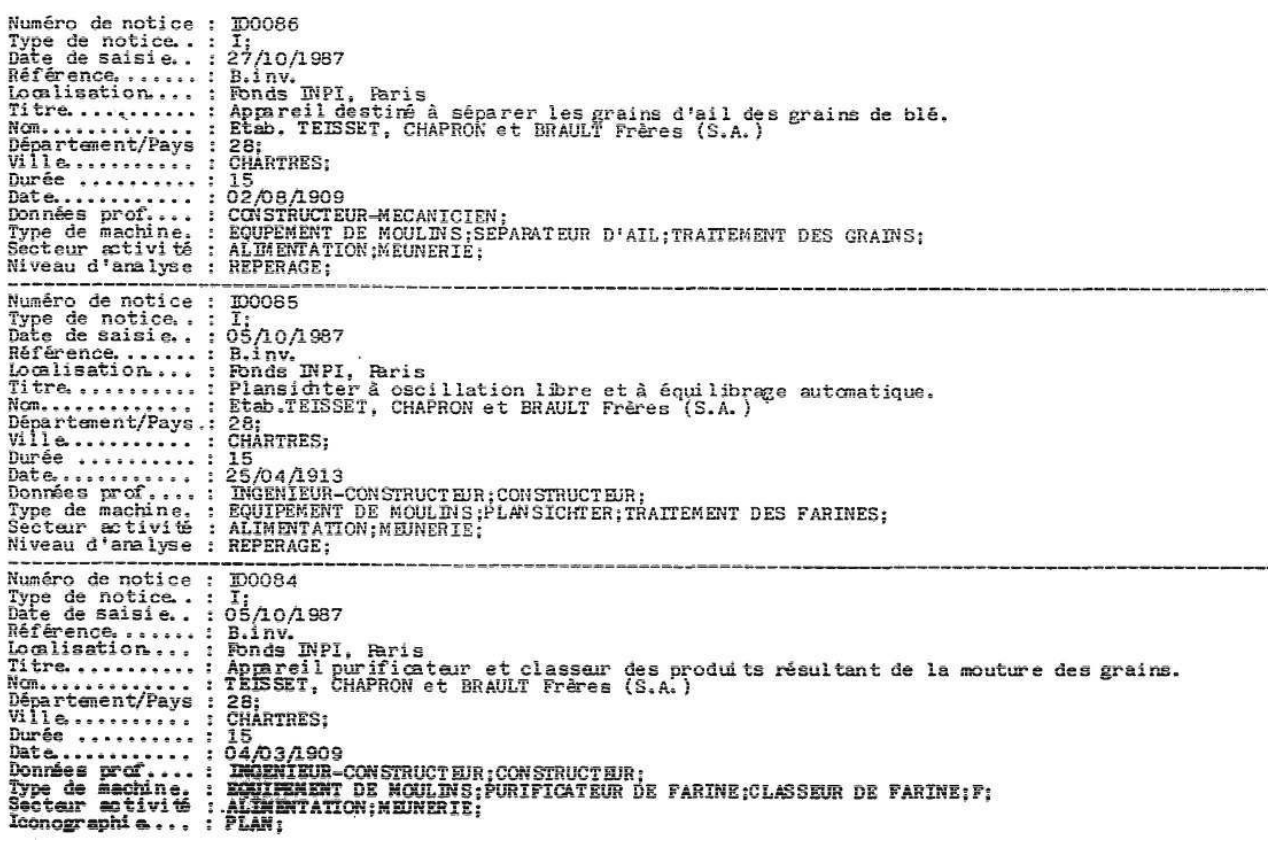


Annexe 4 - L'allure générale des ventes de machines par la S.A.C.M., d'après les livres de commandes

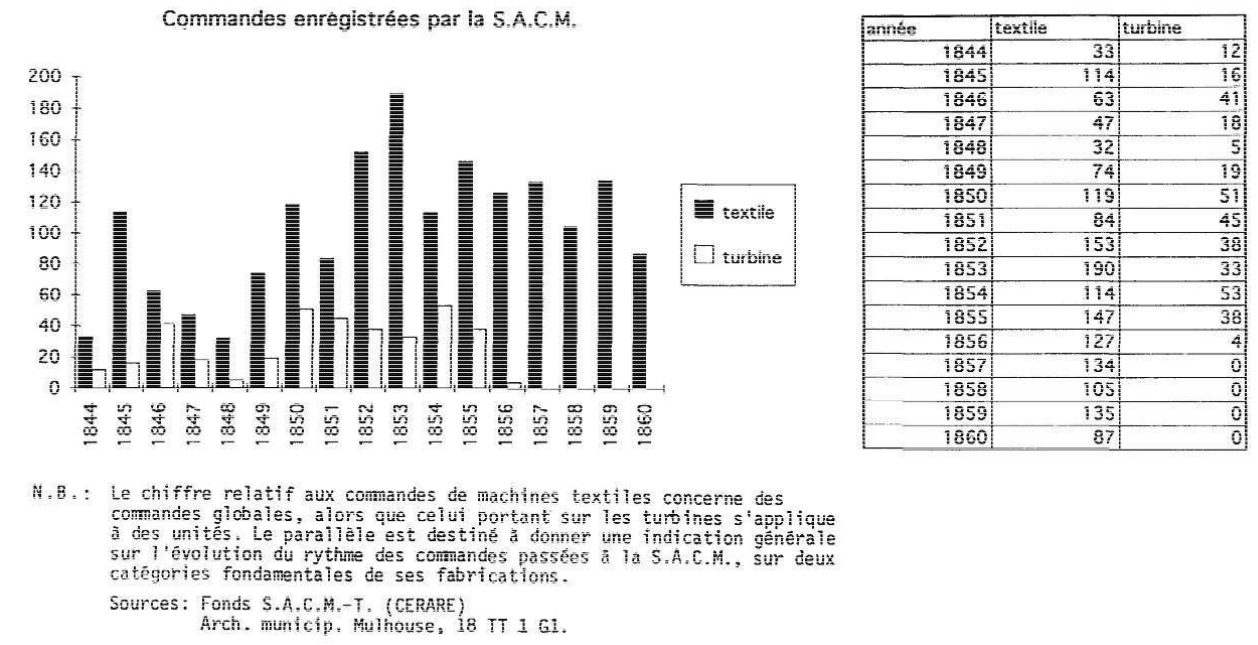

\section{NOTES}

1. Il n'est que de songer, dans cette extension de la mécanisation, à des innovations telles que la photographie, la machine à écrire, ou la machine à calculer mécanique, pour s'en tenir au XIX siècle.

2. Il s'est agi :

$-1^{\circ}$ du programme d'inventaire expérimental des roues hydrauliques, exécuté en 1981-1982, au titre d'un contrat passé avec la MIDIST, doublé d'un second contrat avec la Mission du patrimoine ethnologique au ministère de la culture ;

- 2 de la recherche collective effectuée de 1982 à 1984 au titre du programme Science, technologie et société du CNRS, intitulée Les relations entre conception et réalisation : tradition et innovation dans le domaine des moteurs hydrauliques;

- 30 de l'enquête menée en 1985-1987 dans le cadre de l'Action thématique programmée du CNRS «Histoire industrielle de la France», sur les inventeurs, constructeurs et ingénieurs liés au domaine de l'hydraulique, et portant elle aussi sur le XIX ${ }^{\mathrm{e}}$ siècle.

Ces enquêtes ont notamment donné lieu à la publication de deux recueils d'études, le premier issu des contrats de 1981-1982, et le second des deux recherches suivantes :

- L'Archéologie industrielle en France, n ${ }^{\circ}$ 11, juin 1985 (contributions de L. Bergeron, Cl. Cartier, Cl.-I. Brelot, G. Emptoz et Ph. Peyre, S. Benoit, G. Dufresne, V. Fruit);

- L. Bergeron (sld), «Le moteur hydraulique en France au XIX ${ }^{\mathrm{e}}$ siècle : concepteurs, inventeurs et constructeurs », Cahiers d'histoire et de philosophie des sciences, n ${ }^{\circ} 29$, octobre 1990 (contributions de Br. Belhoste, J.-Fr. Belhoste, S. Benoit, Cl. Cartier, G. Dufresne, G. Emptoz, Cl. Fontanon, L. Lemaître). 
3. S. Benoit, G. Emptoz, Cl. Fontanon, "Une banque de données en développement sur la mécanisation de la France », Cahiers du Centre de recherches historiques, $\mathrm{n}^{\circ}$ 3, avril 1989, p. 15-26.

4. Ce fichier comprend, pour le seul thème des moteurs hydrauliques, quelques 770 brevets retenus par G. Emptoz et Ph. Peyre, sur un total de 1500 relevés à travers les fonds de l'INPI, sur la période 1791-1900 (depuis l'institution, rappelons-le, en 1791 du brevet d'invention en France par l'Assemblée Constituante), une fois éliminés les nombreux brevets en fait étrangers au sujet, tels que ceux proposant explicitement ou implicitement des mouvements perpétuels...

5. Cf. notamment, S. Benoit, «La consommation de combustible végétal et l'évolution des systèmes techniques », dans D. Woronoff, Forges et forêts. Recherches sur la consommation proto-industrielle de bois, Paris, Éditions de l'EHESS, 1990, p. 87-150 ; G. Emptoz, «La carbonisation du bois et la naissance de la chimie du bois: inventions, acteurs et entreprises », Sciences et techniques en perspective, $\mathrm{n}^{\circ} 31,1995$, p. 9-17. Autres publications liées à l'exploitation de ces données à paraître :-S. Benoit et G. Emptoz, «L'actualité du combustible végétal : l'évolution des procédés de carbonisation du bois, $\mathrm{XVIII}^{\mathrm{e}}$-XIX ${ }^{\mathrm{e}}$ siècles », dans D. Woronoff, Techniques, productions et espaces de travail : recherches sur la sidérurgie française dans la première moitié du XIX' siècle.

- G. Emptoz, «Les techniques de carbonisation de la houille en France au cours de la première moitié du XIX ${ }^{\mathrm{e}}$ siècle », dans ibid.

- G. Emptoz, «La locomotive à vapeur et ses combustibles en France au milieu du XIX siècle », in Actes du Congrès international d'histoire des sciences, Saragosse, 22-29 août 1993 (sous presse).

- G. Emptoz, "Chemistry, energy and industrialization : innovation in coke production in France (1800-1870), European Science Foundation, European Workshop, Strategies of chemical industrialization, Université de Liège, 7-8 avril 1994 (sous presse).

6. Sur la sauvegarde et le classement du fonds de la SACM-T, cf. Fl. Ott, « Le sauvetage des archives d'entreprise: l'exemple de la Société alsacienne de constructions mécaniques de Mulhouse (SACM)», pp. 17-23, L'Archéologie industrielle en France, $\mathrm{n}{ }^{\circ} 20-21$, juin 1990, I-Les communications. A propos du fonctionnement du CERARE, cf. Fl. Ott, « Le Centre rhénan d'Archives et de Recherches économiques à l'écoute des problèmes des détenteurs d'archives économiques », ibid., n 17-18, 1989, p. 91-94. Précisons ici que l'intérêt porté, dans le cadre de la banque de données MEDOC à ces deux activités de la SACM mulhousienne, ne saurait faire oublier que l'entreprise eut des activités bien plus diversifiées, à commencer par la fabrication des locomotives (arrêtée à partir de 1889 à l'établissement mulhousien pour être concentrée désormais à celui de Graffenstaden), et celles du matériel électrotechnique, engagée à partir de 1887 à son établissement de Belfort, créé en 1879 et apporté en 1928 à la filiale constituée en commun, sous la raison sociale d'ALSTHOM, par la SACM et la Compagnie française Thomson-Houston.

7. Un décompte manuel, effectué avant la saisie des données relatives au fichier des turbines, avait déjà permis d'établir que plus des $2 / 5$ des ventes de cette catégorie de moteurs (39\% pour le nombre d'unités, $43 \%$ en termes de puissance installée) de la firme mulhousienne, sur les années 1843-1856, avaient été effectuées à l'étranger, notamment en Allemagne, Italie et Suisse. La maison André Koechlin et Cie s'était même, précocement, intéressée au marché nord-américain, comme l'atteste une commande de machines textiles destinée dès 1844 à un établissement de Nouvelle-Angleterre. On sait que, dès 1849, un représentant permanent de la firme, Emile Geyelin, s'établit à Philadelphie, en vue de commercialiser la licence de la turbine Jonval-Koechlin auprès des entreprises de construction mécanique du Nord-Est (cf. S. Benoit, «Les échanges de technologie entre la France et le monde anglo-américain à l'ère de l'industrialisation : le cas des moteurs hydrauliques », Cahiers du Centre de recherches historiques, $\mathrm{n}^{\circ}$ 4, octobre 1989, pp. 15-41.

8. R. Poidevin, «La Société alsacienne de Constructions mécaniques (Mulhouse-Graffenstaden) et le marché français d'après les livres de commandes (1900-1913) », in Actes du Quatre-vingt-douzième 
congrès national des sociétés savantes, Strasbourg et Colmar, 1967, Section d'histoire moderne et contemporaine, II, Le Commerce et l'Industrie, Paris, Bibliothèque nationale, 1970, p. 369-376

9. Arch. munic. Mulhouse, 18 TT 1 G1 à 3. Ces registres ont donné lieu en 1987 à un dépouillement complet sur fiches manuelles sous la direction de Richard Keller. Les résultats de l'exploitation préinformatique qui en a été réalisée par S. Benoit, ont été en partie publiés dans l'article de S. Benoit, G. Dufresne et G. Emptoz, « Une production de pointe dans une entreprise innovante : les turbines de la Maison de Chartres au temps de ses fondateurs (1836-1873)», in L. Bergeron, Le moteur hydraulique en France. Inventeurs, ingénieurs et constructeurs, Cahiers d'Histoire et de Philosophie des Sciences, no 29, octobre 1990, p.151-317 ; et d'autre part dans une note inédite de S. Benoit remise au CERARE, intitulée : Programme de recherche historique sur la Société alsacienne de Constructions mécaniques et la mécanisation internationale de l'industrie textile, novembre 1990.

10. La firme André Koechlin et Cie avait produit des turbines antérieurement à 1843-1844. Elle avait même été l'une des toutes premières firmes, dès 1834, à avoir construit des turbines pour le compte de Benoît Fourneyron au temps où l'illustre inventeur stéphanois n'était encore qu'ingénieur civil. Mais il ne s'agissait pas de turbines conçues par la firme, ce qui est le cas au contraire des machines figurant sur les livres de commandes conservés aux Archives municipales de Mulhouse.

11. Afin de mieux souligner l'articulation entre la recherche menée autour de la banque de données MEDOC et ce séminaire, ainsi que l'approfondissement de ses problématiques, l'intitulé de celui-ci en devient désormais Systèmes énergétiques, mécanisation et industrialisation, XIXe-XXe siècles.

12. Nous renvoyons pour la description de cette première grille à l'article cité supra de $\mathrm{S}$. Benoit, $\mathrm{G}$. Emptoz et Cl. Fontanon paru en avril 1989 dans les Cahiers du CRH.

A ce travail préliminaire de définition, ainsi qu'à la réflexion prospective sur le développement de la banque, ont été associés, sous la direction scientifique de Louis Bergeron : L. André, S. Benoit, B. Belhoste, G. Emptoz, C. Fontanon, A. Grelon, P. Peyre, R. Keller, W. Van Riesen, D. Woronoff.

13. Pour l'installation technique de ce logiciel et sa mise en oeuvre, l'équipe a pu bénéficier de la mise à disposition d'un document de prescriptions très élaboré, sous la forme du cours dispensé sur ce logiciel de bases de données par R. Gimilio, chef du centre de calcul du Groupe de Physique des solides au Département de Physique de l'Ecole Normale Supérieure, dans le cadre d'un cycle d'enseignement assuré en 1987-1988 conjointement avec l'INTD (Institut national des techniques de la documentation) au CNAM. Un manuel de saisie des données et d'utilisation spécifique à la banque MEDOC a été établi par les soins de Marie-Pierre Allix, sous la direction de Jean-Pierre Houillon (INRP) et de Gérard Emptoz (CRH).

Ont également participé comme vacataires à la saisie des fichiers : Mme Amyot, Sylvie de Araujo, Anne-Catherine Hauglustaine-Robert, doctorante à l'EHESS.

14. Cf. l'exploitation faite par G. Emptoz des brevets français concernant les moteurs hydrauliques sur la période 1890-1920, dans la contribution intitulée : «Essor de l'hydroélectricité et construction mécanique en Dauphiné au début du XX⿳⺈冂大 siècle : l'émergence d'un nouveau pôle d'innovation dans le domaine des turbines hydrauliques", in Les scientifiques et la montagne. Actes du 116e Congrès national des Sociétés savantes, Chambéry, 1991, Paris, CTHS, 1993, p. 211-222.

15. Les auteurs de cet article avaient soulevé ce type de problème à propos des ventes de turbines de la firme chartraine fondée par Pierre-Lucien Fontaine, plus important fabricant de ce type d'équipements hydrauliques en France avec la maison André Koechlin et Cie à l'époque du Second Empire, en même temps que premier constructeur français d'équipements de meunerie. Si la firme de Mulhouse, entièrement absente sur ce dernier créneau, ne rivalisait pas sur celui-ci son homologue de Chartres, elle subissait, en revanche, une forte concurrence de la part de cette dernière pour les turbines, auprès des entreprises des secteurs textile, papetier et, à un moindre degré, métallurgique. Cf. S. Benoit, G. Dufresne, G. Emptoz, op. cit., p. 278-302. 